\title{
Transparency and stakeholder engagement in nuclear or radiological emergency management
}

\author{
T. Perko ${ }^{1, *}$, M. Martell ${ }^{2}$ and C. Turcanu ${ }^{1}$ \\ ${ }^{1}$ SCK CEN-Belgian Nuclear Research Centre, Boeretang 200, B-2400 Mol, Belgium. \\ 2 MERIENCE - Strategic Environmental Consultancy, Barcelona, Spain.
}

\begin{abstract}
Stakeholder involvement and transparency are strongly interrelated. Stakeholder involvement is key to strengthening transparency, while transparency is necessary for effective stakeholder involvement. International and European legal requirements in environmental decision-making, radiation protection, nuclear safety and emergency preparedness and response call for increasing levels of transparency and stakeholder engagement. However, recent nuclear or radiological events demonstrate that transparency and engagement in practice are perceived differently by authorities, media and the public. Research conducted in this study by means of a questionnaire sent to authorities responsible for nuclear/radiological emergency management shows a range of challenges related to transparency during a nuclear or radiological emergency, e.g. fear of panic or timely and proper information. Few countries use the full potential of tools and methods for stakeholder engagement in emergency preparedness, although these may also contribute to improved transparency. Despite lessons learned to enhance transparency and stakeholder engagement in nuclear or radiological emergency management, there is room for improvement at a practical level and for finding a common understanding among stakeholders.
\end{abstract}

Keywords: transparency / stakeholders / nuclear emergency / participation / parliament

\section{Introduction}

Stakeholder involvement and transparency are strongly related. Scholars identify transparency as one of the general principles for effective engagement, since transparency acts as an enabler for deliberative and participatory democracy (Rowe et al., 2000; Coleman et al., 2008; Cegarra-Navarro and Garcia-Perez, 2014). Stakeholder engagement is enhanced when stakeholders have the opportunity to participate in (policy) discussions (Bertot et al., 2010), for instance, by developing or contributing to reports on incidents or lessons learned from exercises publicly accessible As stated by the (IAEA, 2017, p.4), "The legitimate concerns of interested parties regarding nuclear and radiation safety matters are best addressed through a culture of transparency and openness, and a strategy to involve, when appropriate, interested parties in decision making".

Research shows that transparency is likely to occur in a context characterized by an active civil society, where alliances are formed between different state and non-state actors, across the branches of government (legislative, executive and judiciary) and involving associations, citizen movements,

*Corresponding author: tanja.perko@sckcen.be civil society organisations, media and academics, etc. (Fox, 2011, p. 29). McGee and Gaventa (2011) point out that engagement of citizens in the "upstream", as well as the "downstream", stages of governance and policy processes shape the impact of transparency and accountability initiatives. While transparency is key to enhancing stakeholder engagement in the governance of nuclear safety (Kerveillant, 2018), the involvement of stakeholders is thus also key to strengthening transparency.

The concept of transparency in nuclear emergency preparedness and response (EP \& R) has vaulted to prominence in recent years. Transparency in EP \& R by nuclear authorities and operators towards different stakeholders is recognised in international documents as essential, and therefore advised and even required (ICRP, 1991; UNEC, 1998; IAEA, 2006, 2007). Within the E.U., new legal requirements comprising provisions for transparency of regulatory authorities and operators of nuclear power plants towards the public have been established. Under the heading of "Transparency" the amended EURATOM Nuclear Safety Directive (Art. 8) requires that "EU Member States shall ensure that necessary information in relation to the regulation of nuclear safety of nuclear installations is made available to the workers and the general public, with specific consideration to local authorities, population and other stakeholders in the 
vicinity of a nuclear installation" (EURATOM, 2014). This obligation "includes ensuring that the competent regulatory authority and the licence holders, within their fields of responsibility, provide in the framework of their communication and stakeholder engagement policy: (a) information on normal operating conditions of nuclear installations to workers and the general public in the fields of its competence; and (b) prompt information in case of incidents and accidents to workers and the general public and to the competent regulatory authorities of other Member States in the vicinity of a nuclear installation." The revised European Basic Safety Standards Council Directive 2013/59/EURATOM of 5 December 2013 also requires Member States to "ensure that information in relation to the justification of classes or types of practices, the regulation of radiation sources and of radiation protection is made available to undertakings, workers, members of the public, as well as patients and other individuals subject to medical exposure." (Art. 77).

The European Basic Standards Directive requires moreover (Art. 70) that "members of the public likely to be affected in the event of an emergency are given information about health protection measures applicable to them and actions they should take in the event of such an emergency", "without any request being made", "updated and distributed at regular intervals and whenever significant changes take place", and permanently available to the public. Furthermore (Art 71), members of the public actually affected are to be "informed without delay about the facts of the emergency, the steps to be taken and, as appropriate, the health protection measures applicable". The European BSS thus focuses on one-way communication, while consultation of stakeholders is mentioned mainly in relation to existing exposure situations (Zeleznik et al., 2019). However, international guidance goes broader and recognises that interested parties should be identified in the preparedness phase and mechanisms should be developed for involving and consulting with relevant interested parties to "enhance the understanding of the complexity of the community, the recognition of the community's capabilities and needs, the fostering of relationships with community leaders, the building and maintaining of partnerships and the empowerment of the local community" (IAEA, 2018). Civil society organisations also request higher involvement in emergency planning at local and national level in European countries and signal a lack of planned involvement of citizens in the EP\& R management itself (NTW, 2015).

Transparent communication is expected to improve trust between stakeholders: "effective stakeholder involvement, continuous communication and a proven track record of transparency during normal operations are important elements for establishing and maintaining public trust at the onset of an emergency" (IAEA, 2012, p. 15). At the same time, transparent communication policy by authorities may trigger additional questions, high intensity media coverage, emotional reactions and heated political discussion (Perko et al., 2012). Past nuclear or radiological events demonstrate that transparency in practice is not perceived in the same way by different EP \& R stakeholders, and the questions "What is transparent?" and "How transparent is transparent enough?" remain open. For instance, after an incident at Krsko Nuclear Power Plant in Slovenia (Perko et al., 2012) authorities claimed they "communicated the event fully transparent" (SNSA, 2008; Stritar, 2009). However, European media reported stakeholders' doubts regarding transparency: "Perhaps there wasn't only a breakdown, but also a huge secret going on?" (Bauer, 2008, p.2); "Europe is on their feet due to the wrong information, perhaps even hiding of the accident." (Jankovič, 2008, p. 5); "What is 'really' going on in the nuclear power plant? What are the Slovenes hiding?" (Pucelj, 2008, p. 1); "only time will show what really happened and what consequences could the breakdown have to our health and the environment." (Il Piccolo, 2008, p. 4).

While the merits of transparency have been linked to trust and credibility, there have been no efforts to investigate how transparency is interpreted and put into practice by authorities in the area of nuclear EP \& R. Similarly, while NEA (2011) investigated methods and channels for interacting with the public among 18 countries worldwide, there has been no systematic investigation among European MS, particularly following the revision of the European BSS. This article identifies in a systematic manner the main challenges faced by regulatory authorities in their efforts to ensure transparency towards stakeholders related to EP\&R. It reviews and discusses European practices in reporting on transparency to the MS' parliaments and in monitoring and reviewing regulatory processes related to EP \& R to ensure openness and transparency to stakeholders. Finally, it investigates the forms of public participation initiated by nuclear regulatory authorities. The manuscript concludes with a critical reflection on transparency and participation practices and proposes research and actions that may support authorities responsible for nuclear or radiological EP \& R to improve their practices.

\section{Method}

Authorities responsible for nuclear emergency management from European MS were contacted to respond to a questionnaire in order to collect their views, among others, on the implementation of international requirements related to transparency and stakeholder engagement in EP \& R. Most of the questions exploring transparency and stakeholder engagement were taken or adapted from NEA (2011). The questionnaire was distributed in the context of a European study on good practices in implementing the requirements on public information in the event of an emergency, under the Euratom BSS Directive and Nuclear Safety Directive (Perko and Martell, 2019). Additional questions related to stakeholder engagement were included in the questionnaire for the ENGAGE project.

The questionnaire was sent on 11 December 2017 to 28 Member States (MS) to at least one e-mail address of the responsible authority for nuclear and radiological emergencies. The questionnaire was sent to more than one institution in most countries, since the responsibility for emergency management and public information tasks related to nuclear or radiological emergencies may be shared between different authorities/organisations (e.g. nuclear safety authority, ministry of internal affairs, ministry of health, national crisis centre, civil protection). Responders were encouraged to collaborate and submit only one response per MS in order to present the situation at national level. However this proved challenging in 
Table 1. Main challenges associated with transparency during a nuclear or radiological emergency in MS. Source of data: Perko and Martell (2019).

\begin{tabular}{ll}
\hline Challenge associated with transparency during a nuclear or radiological emergency & $\begin{array}{l}\text { Countries identifying this challenge associated } \\
\text { with transparency }\end{array}$ \\
\hline Proper information & Hungary, Ireland, Cyprus, Poland, Czech Republic \\
Timeliness of information & Cyprus, Estonia, Spain, Czech Republic \\
Avoiding panic & Austria, Bulgaria, Germany \\
Amount of information & Finland, Poland \\
Reliability of information & Croatia, Italy \\
Avoiding rumours & Sweden \\
Controlled and direct provision of information & Czech Republic \\
Balance between truth and frightening people & France \\
Avoiding confusion, unsettlement, contradictory information & Germany \\
Balance between transparency and reliable communication & \\
Maintain public trust in stress moments and get the main message across & Greece \\
Availability of information & Latvia \\
Security & Croatia \\
Different framework of understanding and identifying public concerns about & Lithuania \\
safety and security issues & \\
Balance between the right to be informed and regulations & Spain \\
Plain language & Poland \\
No particular challenges & Slovak Republic, Slovenia \\
n/a & Malta, Romania \\
/ & Belgium, Denmark, Luxembourg, Netherlands \\
\hline
\end{tabular}

certain MS, and some responses might not be complete or accurate.

Most questions were formulated as open questions and some as statements. Analysis of received responses consisted of three levels. First, responses were reviewed at the moment of the survey reception, pointing out missing responses, inconsistencies, etc and contacting the respondent to clarify the answers. Second, responses were grouped in different categories, classified, and reported as tables. Last, relevant information was deducted from tables to highlight lessons learned, good practices and interesting exceptions. Collection of responses ended on 15 May 2018.

Representatives of authorities from $26 \mathrm{MS}$ responded to the questionnaire. No responses were received from United Kingdom and Portugal for the transparency questions, while Denmark did not provide answers to the engagement questions.

\section{Results}

The majority of MS indicated a wide range of challenges regarding the implementation of transparency during a nuclear or radiological emergency (Tab. 1). Frequently mentioned were avoiding rumours, panic and confusion, as well as the provision of timely, reliable and proper information. Other challenges related, among others, potentially contradictory information, timeliness, sufficiency, clarity, handling the amount of information, keeping the balance between the public's right to be informed and national regulations on emergency planning; between providing the truth and frightening people; and between the desired highest level of transparency and a reliable and deliberate crisis communication. In some countries, the issue of transparency has not been explored yet, and two states reported no particular challenges related to transparency.

As shown in Table 1, the different frameworks for safety and security are a challenge associated with transparency. When asked "What explanations are given to the public on the rationale for what information must be withheld related to an emergency", several countries provided details, e.g. "information on the exact details leading to loss of the sources cannot be released pending the conclusion of the ongoing criminal investigation" (Ireland); or "security related information cannot be public to prevent even worse possible consequences" (Slovenia).

Nine countries stated they report regularly to the parliament on duties concerning transparency related to nuclear emergencies, either through parliamentary questions (Austria, Belgium, Greece, Lithuania), in the framework of the emergency plan (Bulgaria), or as part of the annual reports (Germany, Hungary, Ireland, Lithuania, Romania and Spain). In some cases, e.g. France, there is no systematic procedure, but the nuclear regulatory organisation can be required to report on this topic through parliamentary hearings. 15 out of 25 respondent countries indicated that the responsibility for drafting public information documents concerning nuclear or radiological emergencies and the authority thereof is shared among different actors. 12 countries mentioned they monitor and review their regulatory processes related to EP \& R to ensure openness and transparency, e.g. by means of internal procedures, internal audits, stakeholder consultation, or as part of the nuclear regulatory organisation quality process. Oversight of transparency related to EP \& R is carried out in seven respondent countries: by stakeholders (Austria), by general transparency bodies (Croatia, France, Spain) or by 
Table 2. Methods and channels used routinely by nuclear regulatory authorities to share or exchange information and views with stakeholders related to nuclear emergencies*.

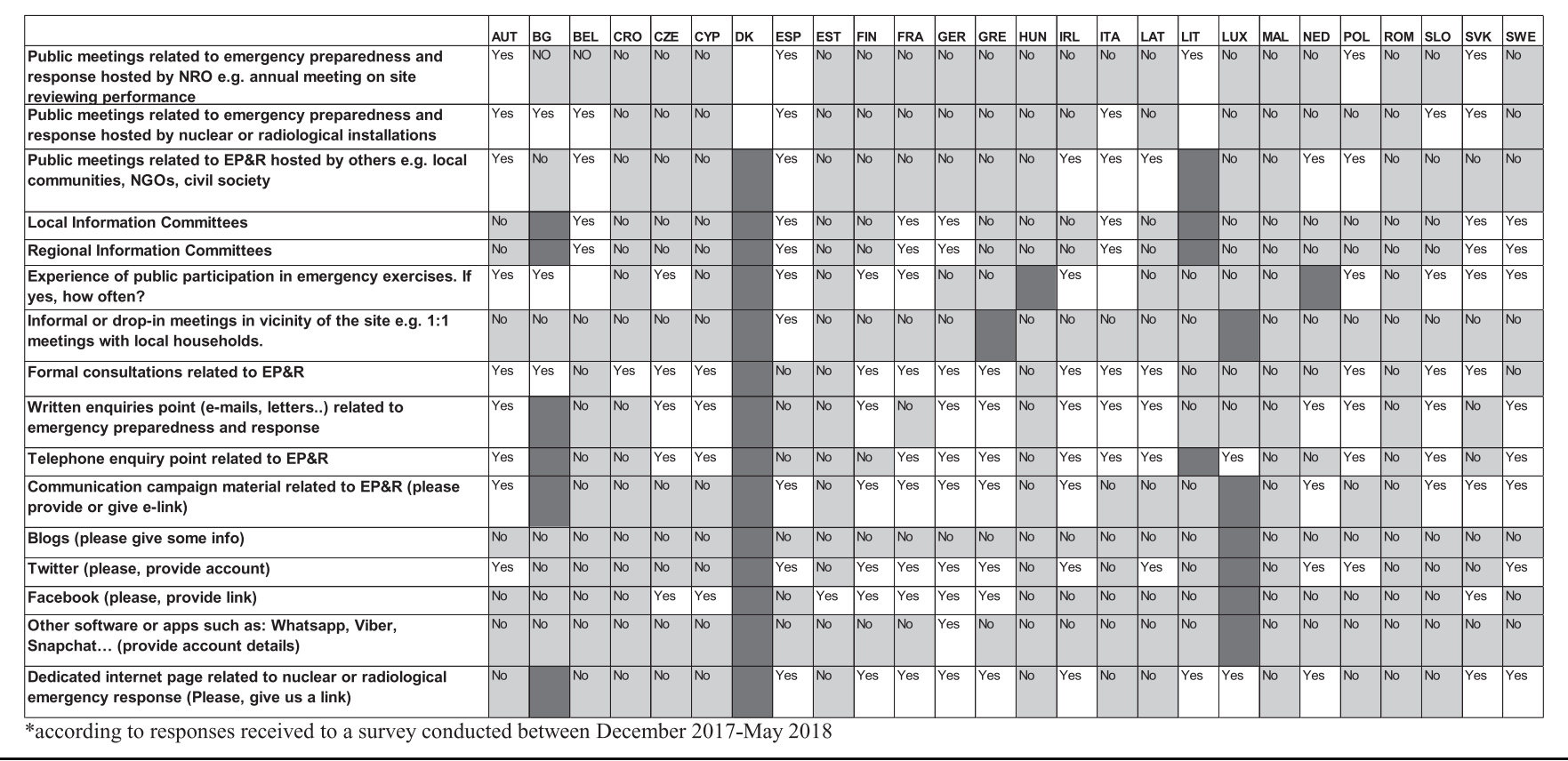

specific governing bodies (Spain, Lithuania, France and Cyprus).

On the topic of public engagement, MS were asked to indicate the methods/channels used routinely by their Nuclear Regulatory Organisation (NRO) "to share or exchange information and views with public/stakeholders related to emergencies". They were asked to differentiate between routine conditions (Tab. 2) and the event of an emergency.

Results suggest that most frequently encountered forms of interactions with the public among MS are formal consultations related to EP \& R, and written and telephone points of enquiry. Participation forms allowing for more regular and active engagement, such as local and regional information committees or public meetings seem less frequently used. Communication through social media (e.g. Twitter and Facebook) also received attention at the time of the survey in several MS. This offers great potential for stakeholder engagement (Perko and Turcanu, 2020). Experiences with public participation in emergency exercises are reported by 11 countries. Examples include: on-line reporting on the NRO's website and through Facebook (Czech Republic), participation in general drills at regular time intervals (Spain, Slovenia, Slovak Republic), frequent participation (Finland, Sweden), participation of members of a national stakeholder panel (Ireland), or participation in exercises at regional level (Poland). Several countries (11 out of 24 respondent countries) reported that their regulatory authorities use dedicated internet pages related to nuclear or radiological emergency response as a channel to share information routinely and exchange views with stakeholders. A number of countries also indicated they have web pages that would become active in the event of an emergency.

\section{Conclusions}

The European Union has developed a legal backdrop requesting transparency to MS (EURATOM, 2013, 2014) and national policies supporting a culture of transparency are established. However, transparency between nuclear safety authorities, operators and other stakeholders is still one of the main concerns in nuclear emergency preparedness, response and recovery (NTW, 2015). The definition of the approach to and the level of transparency is different among the different stakeholders. However, there is a general agreement that the more open and transparent a regulatory body is, the greater chance it has to gain the confidence of stakeholders (NEA, 2011).

Similarly, approaches to stakeholder engagement in EP \& R differ. Commitment to stakeholder engagement from the responsible authorities has been shown to be particularly important to ensure sustainability of these processes (Zeleznik et al., 2019). All MS use some form of interaction with stakeholders. Some countries apply multiple methods and channels for sharing information and exchanging views with stakeholders, including wider publics, while others focus on a limited number, mostly formal consultations on EP \& R and the possibility for enquiry via telephone or in writing. While civil society organisations (NTW, 2015) plead for increased participation in exercises, less than half of the responding MS reported that they had experience with public participation in emergency exercises. Formal consultations and the use of written or telephone enquiry points are reported by a higher number of countries than public meetings organised by the regulator or interactions through local or regional information committees. The results in this respect differ from earlier results by NEA (2011). 
All stakeholders require nuclear regulators and operators to be transparent (NTW, 2015). Our results show that most nuclear regulatory organisations in the European Union strive to give their stakeholders, in particular the public and media, as much digestible information about their role and their decision-making processes as possible. Yet, they are often faced with many challenges, for instance, how to balance security and safety concerns or how to avoid panic and give proper, accurate and timely information. Although nuclear regulatory authorities understand that enhancing transparency serves to give the public and other stakeholders confidence in their role of protecting public health and the environment and ensuring public safety and security, they have concerns to find the right balance between saying the truth and frightening people. The implementation of transparency practices in each MS differs depending on the cultural context, stakeholder engagement practices or the availability (lack) of the necessary resources. A majority of countries have no special procedures for monitoring and reviewing transparency of nuclear safety authorities to stakeholders.

Several MS indicated the existence of committees or governing bodies responsible for the oversight of transparency related to EP \& R, e.g. oversight is done by stakeholders, general transparency bodies or specific governing bodies. This practice was found only in eight MS out of 26 respondent countries, but this type of structure could play an important role in promoting transparency. In addition, monitoring and reviewing the regulatory processes related to EP \& R is a good practice to ensure openness and transparency.

As pointed out, transparency, accountability and public engagement are strongly inter-connected. Strengthening participation in the preparedness for emergency response and recovery post-accident may increase the trust in the responsible authorities and lead to improved perception of their transparency. Conducting empirical research on the relationship between stakeholder engagement and transparency is recommended.

Whilst there are different practices in different European countries, there is no common understanding on what is transparency in practice in EP \& R and there are no specific guidelines on how to improve transparency in practice. In order to improve understanding of transparency and processes related to transparent communication between different stakeholders, co-developing dedicated practical guidelines among the different authorities and stakeholders related to nuclear or radiological emergencies may contribute to improving practice and meet different stakeholders' expectations.

Acknowledgments. This work received funding from: (i) "Study on good practices in implementing the requirements on public information in the event of an emergency, under the Euratom Basic Safety Standards Directive and Nuclear Safety Directive" supported by DG Energy, Ares (2016) 7037963; and (ii) ENGAGE project, which is part of $\mathrm{H} 2020$ CONCERT; this project has received funding from the EURATOM research and training programme 2014-2018 under grant agreement No. 662287.

Disclaimer (Art. 29.5 GA). This publication reflects only the author's view. Responsibility for the information and views expressed therein lies entirely with the authors. The European Commission is not responsible for any use that may be made of the information it contains.

\section{References}

Bauer M. 2008. Obrazec. Slovenske novice 2.

Bertot JC, Jaeger PT, Grimes JM. 2010. Using ICTs to create a culture of transparency? E-government and social media as openness and anti-corruption tools for societies. Govern. Inform. Q. 27: 264-271.

Cegarra-Navarro J, Garcia-Perez A. 2014. Technology knowledge and governance: Empowering citizen engagement and participation. Govern. Inform. Q. 31(4): 660-668.

Coleman R, Lieber P, Mendelson AL, Kurpius DD. 2008. Public life and the internet: If you build a better website, will citizens become engaged? New Media Soc. 10(2): 179-201.

EURATOM. 2013. Council Directive 2013/59/EURATOM of 5 December 2013. Basic safety standards for protection against the dangers arising from exposure to ionising radiation.

EURATOM. 2014. Nuclear safety directive (2014). 2014/87/ Euratom.

Fox J. 2011. Government transparency and policymaking. Public Choice 131(1-2): 23-44.

IAEA, International Atomic Energy Agency. 2006. Fundamental safety principles. Vienna, Austria: IAEA Safety Standards 2006, SF-1.

IAEA. 2007. Safety glossary: Terminology used in nuclear safety and radiation protection. Vienna.

IAEA. 2012. Enhancing transparency and communication effectiveness in the event of a nuclear or radiological emergency. In: Int. Exp. Meeting 18-20 June, 2012, Vienna, Austria.

IAEA. 2017. Communication and consultation with interested parties by the regulatory body. IAEA Safety Standards Series No. GSG-6. English STI/PUB/1784.

IAEA. 2018. Arrangements for the termination of a nuclear or radiological emergency. IAEA Safety Standards for protecting people and the environment. General Safety Guide No. GSG-11.

ICRP. 1991. Recommendation of the International Commission on Radiological Protection, Vol.60. Oxford, New York, Frankfurt, Seoul, Sydney, Tokyo: Protection ICOR, Ed. Pergamon Press.

Il Piccolo. 2008. Metz: "Un episodo da non sottovaluare". Il Piccolo 4.

Jankovič J. 2008. JEK ustavljena. Slovenske novice 5.

Kerveillant M. 2018. The role of the public in the French nuclear sector: The case of "Local Information Commissions" (CLIs) for nuclear activities. Radioprotection 53(2): 87-93.

McGee R, Gaventa J. 2011. Shifting power? Assessing the impact of transparency and accountability initiatives. IDS Working Papers 2011(383): 1-39.

NEA. 2011. Commendable practices on transparency in nuclear regulatory communication with the public. Nuclear Energy Agency. Available from https://www.oecd-nea.org/nsd/docs/ 2011/cnra-r2011-3.pdf.

NTW. 2015. Report of NTW Working Group on Emergency Preparedness \& Response $(E P \& R)$. Available from http:// www.nuclear-transparency-watch.eu/wp-content/uploads/2015/ 04/NTW-Report.pdf.

Perko T, Martell M. 2019. Study on good practices in implementing the requirements on public information in the event of an emergency, under the Euratom Basic Safety Standards Directive and Nuclear Safety Directive. Final report.

Perko T, Turcanu C. 2020. Is internet a missed opportunity? Evaluating radon websites from a stakeholder engagement perspective. J. Env. Rad. 212: 106-123.

Perko T, Turcanu C, Carlé B. 2012. Media reporting of nuclear emergencies: The effects of transparent communication in a minor nuclear event. J. Cont. Crisis Manag. 20: 52-56. 
Pucelj G. 2008. Vaja. Delo 1.

Rowe G, Frewer L, Sjöberg L. 2000. Newspaper reporting to hazard in the UK and Sweden. Public Understanding Sci. 9: 59-78.

SNSA. 2008. Description of the event at the Krško NPP on 4 June 2008. News. Slovenian Nuclear Safety Administration.

Stritar A. 2009. Incident at Krško NPP and panic in Europe. In: PIME, 15-18 Feb., 2009, Edinburgh.
UNEC. 1998. Aarhus Convention on Access to information, public participation in decision-making and access to justice in environmental matters. Europe, UNEC: Ed. Aarhus.

Zeleznik N et al. 2019. Report on venues, challenges, opportunities and recommendations for stakeholder engagement in emergency and recovery preparedness and response. CONCERT Deliverable D9.90.

Cite this article as: Perko T, Martell M, Turcanu C. 2020. Transparency and stakeholder engagement in nuclear or radiological emergency management. Radioprotection 55(HS2): S243-S248 